\title{
Use of partial data from failed adrenal venous sampling to guide surgical management in primary aldosteronism; a case report and review of literature
}

\author{
De Silva N. L1 ${ }^{1}$, Ganewatte E ${ }^{2}$, Appuhamy $C^{3}$, Sumanatilleke $M^{4}$ \\ ${ }^{1}$ Department of Clinical Sciences, Faculty of Medicine, General Sir John Kotelawala Defence University \\ ${ }^{2}$ Department of Radiology, Lady Ridgeway Hospital \\ ${ }^{3}$ Department of Radiology, Colombo North Teaching Hospital \\ ${ }^{4}$ Diabetes and Endocrine Unit, National Hospital of Sri Lanka
}

\section{Abstract}

Primary aldosteronism is associated with increased morbidity, mortality, and poor quality of life. Attempts should be made to diagnose, establish subtype classification, and offer the most appropriate diagnosis depending on the subtype. Adrenal venous sampling is the gold standard method in subtype classification. However, failure to cannulate the right adrenal vein is commonly encountered. A 55-year-old male with hypertension for 21 years, presented with generalized body aches, paraesthesia, and cramps for three months. His blood pressure was controlled on telmisartan and amlodipine. Serum potassium was $2.9 \mathrm{mmol} / \mathrm{L}$. Serum aldosterone was elevated with suppressed plasma renin concentration. Magnetic resonance imaging of the abdomen showed a well-defined round $1.4 \mathrm{~cm} \times 1.3$ $\mathrm{cm}$ isointense lesion in the right suprarenal gland and multiple bilateral renal cysts. During bilateral adrenal vein sampling, the right adrenal vein was not cannulated. Aldosterone: cortisol ratio of the left adrenal vein and inferior vena cava were 0.0264 and 0.0699 , respectively. Left adrenal vein/ inferior vena cava index was 0.377 , suggesting contralateral aldosterone excess. He underwent laparoscopic right adrenalectomy, which resulted in the resolution of hypertension and hypokalaemia. Careful analysis of partial data from 'failed' adrenal vein sampling can support management decisions avoiding unnecessary repetition of invasive procedures.

Key Words: primary aldosteronism, adrenal venous sampling, unilateral catheterization

Correspondence email: nipunlak@gmail.com

https://orcid.org/0000-0002-4467-3659

This is an open-access article distributed under the terms of the Creative Commons Attribution License, which permits unrestricted use, distribution, and reproduction in any medium, provided the original author and source are credited (CC BY 4.0)

\section{Introduction}

Primary aldosteronism is the commonest secondary cause of hypertension (1). In addition to complications of hypertension, it is associated with aldosterone-specific cardiovascular and renal morbidity and mortality. poor quality of life. Therefore, diagnosis and optimal management of this condition would improve patient outcomes beyond blood pressure control. Improvement of hypertension and hypokalaemia is likely to occur in most patients with appropriate management, though cure of hypertension is seen only in $30-60 \%$ of the patients (1).

In the management of primary aldosteronism, one of the most important steps is the subtype classification between unilateral aldosterone-producing adenoma (APA) and bilateral idiopathic hyperaldosteronism (IHA). Unilateral APA is treated by ad- 
renalectomy, whereas bilateral IHA is treated medically using mineralocorticoid receptor antagonists. Adrenal imaging using computed tomography (CT) and magnetic resonance imaging (MRI) performs poorly in differentiating between two main sub- types $(2,3)$. Except in individuals younger than 35 years, this helps localization in less than $60 \%$ of the patients. As a result, bilateral adrenal venous sampling (AVS) has become the mainstay of subtype classification.

\section{Table 1 Summary of investigations at the time of the presentation}

\begin{tabular}{|c|c|c|}
\hline Investigation & Result & Reference range \\
\hline Haemoglobin $(\mathrm{g} / \mathrm{dL})$ & 13.9 & $11-16$ \\
\hline Mean corpuscular volume (fl) & 87.1 & $80-96$ \\
\hline White cell count $\left(\times 10^{9} / \mathrm{L}\right)$ & 8.2 & $4-11$ \\
\hline Platelet count $\left(\times 10^{9} / \mathrm{L}\right)$ & 375 & $150-450$ \\
\hline Urinalysis & Normal & \\
\hline Serum creatinine $(\mathrm{mg} / \mathrm{dL})$ & 1.02 & $0.7-1.2$ \\
\hline Sodium $(\mathrm{mmol} / \mathrm{L})$ & 140 & $135-145$ \\
\hline Potassium (mmol/L) & 2.9 & $3.5-5.1$ \\
\hline $\begin{array}{l}\text { Spot urinary potassium } \\
(\mathrm{mmol} / \mathrm{L})\end{array}$ & 22.5 & $>20$ suggestive of renal loss \\
\hline Plasma bicarbonate (mEq/L) & 35 & $22-28$ \\
\hline Aspartate transaminase $(\mathrm{U} / \mathrm{L})$ & 19 & $<40$ \\
\hline Alanine transaminase $(\mathrm{U} / \mathrm{L})$ & 34 & $<40$ \\
\hline Bilirubin (mg/dL) & 0.7 & $0.5-1.1$ \\
\hline $\mathrm{HbA1c}$ & $5.6 \%$ & $<5.7 \%$ \\
\hline $\begin{array}{l}\text { Thyroid-stimulating hormone } \\
(\mathrm{mIU} / \mathrm{l})\end{array}$ & 0.87 & $0.5-4.7$ \\
\hline $\begin{array}{l}\text { Twenty-four-hour urinary } \\
\text { metanephrine }(\mu \mathrm{g} / \mathrm{dL})\end{array}$ & 217 & $<350$ \\
\hline Aldosterone (ng/dL) & 37 & $1.76-23.2$ \\
\hline $\begin{array}{l}\text { Plasma renin concentration } \\
(\mathrm{mIU} / \mathrm{mL})\end{array}$ & 1.98 & $2.8-39.9$ \\
\hline Aldosterone: renin ratio & 18.64 & $<3.7$ \\
\hline
\end{tabular}


Successful cannulation of both adrenal veins is technically demanding and requires a high degree of expertise. Cannulation of the right adrenal vein is particularly difficult. Though experienced centers have reported successful cannulation rates of both sides in up to $96 \%$ (3), failure to cannulate the right side can be seen in more than $30 \%$ in low volume centers $(4,5)$. Therefore, failure to cannulate one side can limit the benefit of this invasive procedure. Repeat testing would increase the cost, radiation exposure, and contrast agents.

Therefore, it is imperative to try and interpret partial data from these 'failed' procedures while maintaining the accuracy of the clinical decision.

Though the cannulation of the right side was not successful in our patient described below, careful assessment of the data allowed successful surgery for primary aldosteronism.

\section{Case Report}

A 55-year-old lawyer presented with generalized body aches, paraesthesia, and cramps for three months. Symptoms were slowly progressing over time without associated fever, muscle weakness, or loss of weight or appetite.
He was diagnosed with hypertension 21 years back. Since then, blood pressure control was satisfactory with two antihypertensive agents. At the time of the presentation, he was on telmisartan and amlodipine. Over the last few months, blood pressure control had not been achieved. There were no other co-morbidities. He had undergone left meniscectomy 25 years back and tonsillectomy five years back. At the time of tonsillectomy, serum potassium was $3.4 \mathrm{mmol} / \mathrm{L}$. This had not been further pursued at that time. He has never smoked, and he is a teetotaller.

While being evaluated, he was found to have hypokalaemia $(2.9 \mathrm{mmol} / \mathrm{L})$. He was not on any other drugs in addition to the above antihypertensive agents. There was no polyuria, altered bowel habits, or diarrhea. He was started on oral potassium chloride. Potassium normalized to $3.9 \mathrm{mmol} / \mathrm{L}$. Results of the laboratory investigations performed during the presentation are summarised in Table 1.

In the presence of hypertension, hypokalaemia, and metabolic alkalosis, a presumptive diagnosis of primary aldosteronism was made.

Aldosterone renin ratio was performed while on telmisartan, amlodipine, and oral KCL. Though

\section{Figure 1 Isointense lesion in the right suprarenal gland}

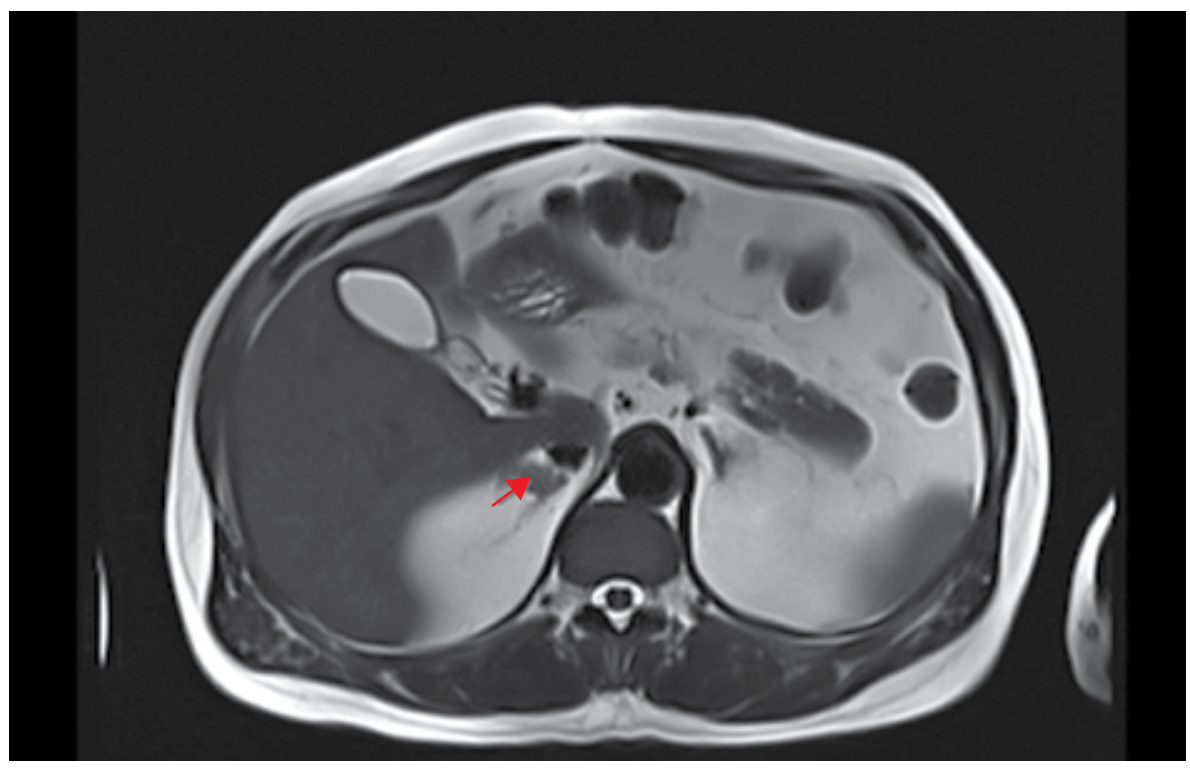

T2 weighted axial MRI of the abdomen showing a well-defined iso-intense lesion in the right adrenal gland measuring $1.4 \times 1.3 \mathrm{~cm}$. There is a clear signal drop-out in the out of phase imaging indicating fat within the lesion. There are no calcifications. The left adrenal gland appears normal. 
telmisartan can potentially produce false-negative results, it was not discontinued due to persistent hypokalaemia while taking it. Due to the presence of aldosterone level $>20 \mathrm{ng} / \mathrm{dL}$, renin lower than the reference range, and spontaneous hypokalaemia, it was considered that he does not need confirmatory testing $(6,7)$.

The diagnosis of primary aldosteronism was made. Magnetic resonance imaging of the abdomen showed a well-defined rounded $1.4 \mathrm{~cm} \times 1.3 \mathrm{~cm}$ isointense lesion in the right suprarenal gland. The left adrenal gland appeared normal (Figure 1).

There were multiple bilateral renal cysts. Renal cysts are known to occur in patients with primary aldosteronism (8).
He underwent unstimulated AVS after changing medicines to extended-release prazosin and sustained-release verapamil.

Images acquired during the procedure are shown in figure 2. The results of the study are shown in Table 2.

Findings were suggestive of a lack of successful cannulation of the right adrenal vein. But following calculation was performed using the available data.

Aldosterone/ cortisol ratio of left adrenal vein $(\mathrm{LAV})=0.0264$

Aldosterone/ cortisol ratio of inferior vena cava $(\mathrm{IVC})=0.0699$

LAV/IVC index: $0.0264 / 0.0699=0.377$

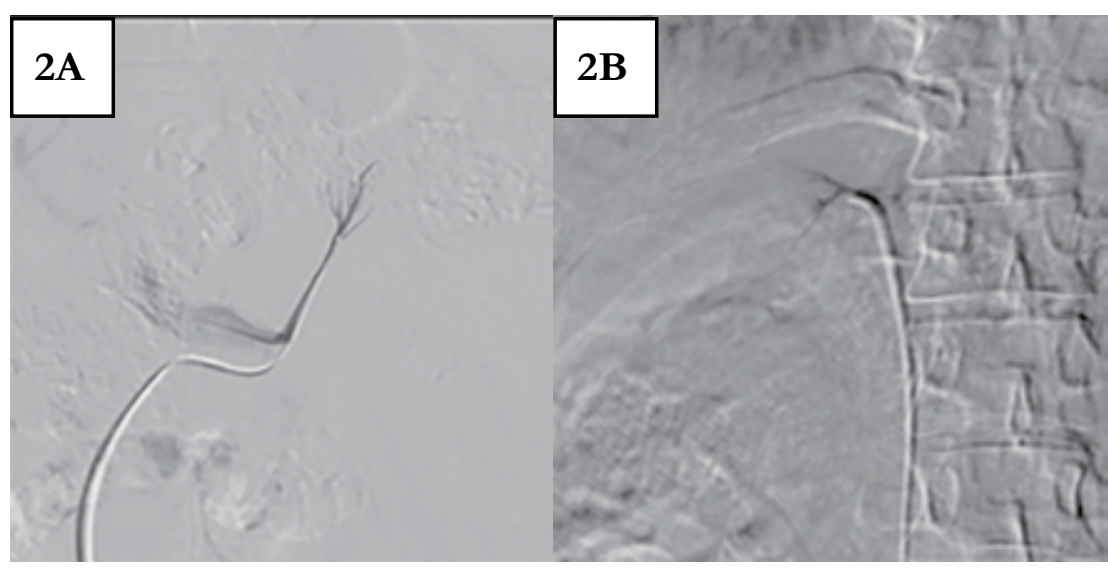

Figure 2 Fluoroscopic images were acquired during catheterization of the left (Figure 2A) and presumed right (Figure $2 \mathrm{~B}$ ) adrenal veins

Table 2 Results of bilateral adrenal vein sampling

\begin{tabular}{cccc}
\hline Site & Cortisol (nmol/L) & $\begin{array}{c}\text { Aldosterone } \\
\text { (ng/dL) }\end{array}$ & $\begin{array}{c}\text { Selectivity index } \\
\text { (adrenal vein } \\
\text { cortisol/ IVC } \\
\text { cortisol) }\end{array}$ \\
\hline Left adrenal vein & 905 & 23.9 & 3 \\
Right adrenal vein & 291 & 22.7 & 0.98 \\
Inferior vena cava & 296 & 20.7 & N/A \\
\hline
\end{tabular}

*IVC- inferior vena cava 
Since AV/IVC index $<0.5$ suggests unilateral disease from the opposite side, he underwent laparoscopic right adrenalectomy (9). Aldosterone dropped to $2.59 \mathrm{ng} / \mathrm{dL}$ one day after surgery. Within two weeks, blood pressure became normal without any antihypertensive agent. One week after surgery, serum potassium was $4.2 \mathrm{mmol} / \mathrm{L}$ without replacement.

One month after surgery, serum creatinine was 1.8 $\mathrm{mg} / \mathrm{dL}$ with an eGFR of $41.7 \mathrm{ml} / \mathrm{min} / 1.73 \mathrm{~m} 2$. This decline in eGFR after surgery is likely due to relative hyperfiltration seen in primary aldosteronism (10). Serum creatinine stabilized thereafter with no further increase. Histology confirmed completely resected $10 \mathrm{~mm}$ right adrenal cortical adenoma. Currently, the patient is asymptomatic and feels well.

\section{Discussion}

Bilateral AVS is the gold standard method of subtype classification in primary aldosteronism. In the Sri Lankan setting, sequential catheterization of left and right adrenal veins was performed. Samples are obtained for cortisol and aldosterone levels. Same samples are obtained from the inferior vena cave or femoral vein as well. Successful cannulation is defined using the selectivity index. An unstimulated AVS selectivity index of $\geq 2$ suggests successful cannulation of the given adrenal vein (5). When both sides are cannulated, the lateralization index is calculated by dividing the aldosterone/ cortisol ratio of the higher side from that of the lower side. Value $\geq 4$ is commonly used to define unilateral APA (11).

The major limitation of interpreting AVS data is the failure to cannulate the right side. Failed right side cannulation is a common occurrence, particularly in low volume centers $(4,5)$. This is due to multiple reasons, including the short length, small caliber, and angulation at the origin of the right adrenal vein. In addition, it can arise in conjunction with hepatic vein branches, and it can be confused with small hepatic vein branches which lie nearby (11). The lateralization index cannot be calculated when one side is not calculated. As a result, traditionally, these patients had to undergo repeat AVS increase cost, radiation, and contrast agent exposure.

In such a scenario use of partial data from the successful left side can be evaluated carefully to guide management. Some studies have shown that suppressed cortisol corrected aldosterone levels in the cannulated adrenal vein compared to that of the IVC indicates contralateral APA (12). This had been systematically studied using 36 patients in the derivation cohort and 26 patients in the validation cohort. They have concluded that LAV/IVC aldosterone: cortisol ratio (AV/IVC index) $<0.5$ would reliably confirm contralateral aldosterone success. Similarly, a value $>5.5$ would reliably suggest ipsilateral disease (9). Values in between would require repeat AVS. Using their data, authors have indicated that this approach would prevent $50 \%$ repeat AVS procedures.

This was re-examined using simulated unilateral data from 150 patients who had successful bilateral AVS (13). By comparing unilateral data with the final diagnosis, they have confirmed that AV/IVC index $<0.5$ confirms contralateral unilateral disease. Using this cut-off will not lead to any inappropriate adrenalectomies though some contralateral disease will be missed. However, according to their data, it was shown that using AV/IVC index $>5.5$ would lead to inappropriate adrenalectomies in $18 \%$ since those patients have had bilateral disease. One limitation in applying the data from the above two studies to our patient is the lack of cosyntropin stimulation in him. Both studies have defined cut-offs using data from patients who received cosyntropin stimulation. Due to the lack of high-quality data on such patient populations, we had to depend on the assumption that markedly suppressed aldosterone in the contralateral adrenal vein would reliably exclude bilateral disease irrespective of cosyntropin stimulation. A successful outcome in our patient, confirmed biochemically and by resolution of hypertension and hypokalaemia despite long-standing disease, strengthens our approach.

\section{Conclusions}

While it is well established that an experienced interventionist must perform AVS to improve the results of the procedure, it should be interpreted by 
an experienced clinician who can utilize data from even 'failed AVS' minimizing unnecessary repeat procedures and adrenalectomies.

\section{List of abbreviations}

APA- aldosterone-producing adenoma

$\mathrm{AV}$ - adrenal vein

AVS- adrenal venous sampling

CT- Computed tomography

eGFR- estimated glomerular filtration rate

IHA- Idiopathic hyperaldosteronism

IVC- inferior vena cava

MRI- Magnetic resonance imaging

\section{References}

1. Young WF. Primary aldosteronism: renaissance of a syndrome. Clinical endocrinology. 2007;66(5):60718.

2. Lim V, Guo Q, Grant CS, Thompson GB, Richards ML, Farley DR, et al. Accuracy of adrenal imaging and adrenal venous sampling in predicting surgical cure of primary aldosteronism. The Journal of Clinical Endocrinology \& Metabolism. 2014;99(8):2712-9.

3. Young Jr WF, Stanson AW, Thompson GB, Grant CS, Farley DR, Van Heerden JA. Role for adrenal venous sampling in primary aldosteronism. Surgery. 2004;136(6):1227-35.

4. Siracuse JJ, Gill HL, Epelboym I, Clarke NC, Kabutey N-K, Kim I-K, et al. The vascular surgeon's experience with adrenal venous sampling for the diagnosis of primary hyperaldosteronism. Annals of vascular surgery. 2014;28(5):1266-70.

5. Vonend O, Ockenfels N, Gao X, Allolio B, Lang $\mathrm{K}$, Mai $\mathrm{K}$, et al. Adrenal venous sampling: evaluation of the German Conn's registry. Hypertension. 2011;57(5):990-5.
6. Young Jr W. Diagnosis and treatment of primary aldosteronism: practical clinical perspectives. Journal of internal medicine. 2019;285(2):126-48.

7. Funder JW, Carey RM, Mantero F, Murad MH, Reincke M, Shibata H, et al. The management of primary aldosteronism: case detection, diagnosis, and treatment: an endocrine society clinical practice guideline. The Journal of Clinical Endocrinology \& Metabolism. 2016;101(5):1889-916.

8. Torres VE, Young Jr WF, Offord KP, Hattery RR. Association of hypokalemia, aldosteronism, and renal cysts. New England Journal of Medicine. 1990;322(6):345-51.

9. Pasternak JD, Epelboym I, Seiser N, Wingo M, Herman M, Cowan V, et al. Diagnostic utility of data from adrenal venous sampling for primary aldosteronism despite failed cannulation of the right adrenal vein. Surgery. 2016;159(1):267-74.

10. Ribstein J, Du Cailar G, Fesler P, Mimran A. Relative glomerular hyperfiltration in primary aldosteronism. Journal of the American Society of Nephrology. 2005;16(5):1320-5.

11. Young WF, Stanson AW. What are the keys to successful adrenal venous sampling (AVS) in patients with primary aldosteronism? Clinical endocrinology. 2009;70(1):14-7.

12. Wolley MJ, Gordon RD, Ahmed AH, Stowasser M. Does contralateral suppression at adrenal venous sampling predict outcome following unilateral adrenalectomy for primary aldosteronism? A retrospective study. The Journal of Clinical Endocrinology \& Metabolism. 2015;100(4):1477-84.

13. Strajina V, Al-Hilli Z, Andrews JC, Bancos I, Thompson GB, Farley DR, et al. Primary aldosteronism: making sense of partial data sets from failed adrenal venous sampling-suppression of adrenal aldosterone production can be used in clinical decision making. Surgery. 2018;163(4):801-6. 